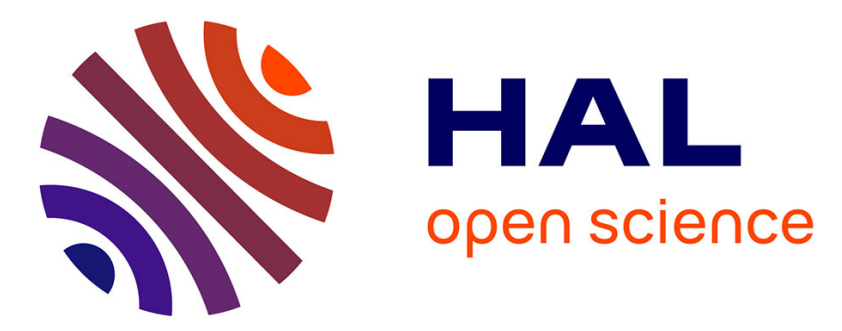

\title{
Cinétiques comparées des dégradations dans le sol du 2,4-D et du 2,4-dichlorophénol seuls ou en mélange. Conséquences sur le comportement des biomasses microbiennes dégradantes correspondantes
}

\author{
Guy Soulas, Jean-Claude Fournier, Nadine Rouard
}

\section{To cite this version:}

Guy Soulas, Jean-Claude Fournier, Nadine Rouard. Cinétiques comparées des dégradations dans le sol du 2,4-D et du 2,4-dichlorophénol seuls ou en mélange. Conséquences sur le comportement des biomasses microbiennes dégradantes correspondantes. Agronomie, 1987, 7 (3), pp.193-199. hal00884984

\author{
HAL Id: hal-00884984 \\ https://hal.science/hal-00884984
}

Submitted on 1 Jan 1987

HAL is a multi-disciplinary open access archive for the deposit and dissemination of scientific research documents, whether they are published or not. The documents may come from teaching and research institutions in France or abroad, or from public or private research centers.
L'archive ouverte pluridisciplinaire HAL, est destinée au dépôt et à la diffusion de documents scientifiques de niveau recherche, publiés ou non, émanant des établissements d'enseignement et de recherche français ou étrangers, des laboratoires publics ou privés. 


\section{Cinétiques comparées des dégradations dans le sol du 2,4-D et du 2,4-dichlorophénol seuls ou en mélange. Conséquences sur le comportement des biomasses microbiennes dégradantes correspon- dantes}

Guy SOULAS \& Jean-Claude FOURNIER

avec la collaboration technique de Nadine RouARD

I.N.R.A., Laboratoire de Microbiologie des Sols, Centre de Recherches de Dijon, 17, rue Sully, F 21034 Dijon Cedex

Le 2,4-dichlorophénol (2,4-DCP) étant un produit de dégradation du 2,4-D, nous avons cherché à analyser la cinétique de cette transformation en étudiant les modifications de la courbe de dégradation de l'un des deux composés précédents en présence de différentes concentrations de l'autre. Pour cela, nous avons utilisé des produits marqués au ${ }^{14} \mathrm{C}$ et suivi leur dégradation par l'intermédiaire du dégagement de ${ }^{14} \mathrm{CO}_{2}$. Nous avons en outre, étudié les conséquences des traitements précédents sur l'état d'activité et la taille de la microflore dégradant le 2,4-D. Nous avons montré - 1) que le 2,4-DCP est probablement un substrat potentiellement plus facilement utilisable par les micro-organismes du sol que le 2,4-D - 2) que le 2,4-DCP directement ajouté au sol y est rapidement et fortement fixé -3) que cette dernière propriété le rend peu accessible aux micro-organismes. Il interfère donc peu avec le 2,4-DCP produit, à partir de 2,4-D, dans des cellules microbiennes où il n'est souvent qu'un intermédiaire transitoire.

Mots clés additionnels : Adaptation, fumigation, phase de latence.

Breakdown kinetics of 2,4-D and 2,4-dichlorophenol in soil, alone or in mixture, and effects on the behaviour of the respective degrading microbial biomass.

2,4-dichlorophenol (2,4-DCP) is a main degradation product of 2,4-D in the soil. We analysed kinetics of this transformation by studying modifications in the shape of the degradation curve of one compound at different concentrations of the other. Kinetics of breakdown were followed by ${ }^{14} \mathrm{CO}_{2}$ evolution from ${ }^{14} \mathrm{C}$-labelled chemicals. Moreover we studied the consequences of these treatments on the activity level and size of the 2,4-D degrading microflora. The main conclusions were : 1) $2,4-\mathrm{DCP}$ is probably a potentially more easily mineralizable substrate for soil microorganisms than is 2,4-D ; 2) 2,4-DCP directly added to the soil is readily and firmly bound to some soil components ; 3 ) this property makes it not easily available for microorganisms. There is accordingly little interaction with the 2,4 -DCP produced from $2,4-\mathrm{D}$ within microbial cells, where it is often an intermediary compound.

Additional key words : Adaptation, fumigation, lag-phase.

\section{INTRODUCTION}

Le nombre de pesticides qui peuvent être utilisés comme source de $\mathrm{C}$ et d'énergie par certaines souches de micro-organismes du sol est relativement limité. Plus souvent, la minéralisation de ces composés nécessite l'intervention de plusieurs souches microbiennes possédant des capacités métaboliques complémentaires. Il apparaît donc, au cours de la dégradation, des produits de transformation (métabolites) qui peuvent s'accumuler de façon plus ou moins transitoire d'abord à l'intérieur de la cellule des espèces dégradantes puis dans le sol. Cette accumulation des métabolites peut être à l'origine d'une.modification de la cinétique 
de dégradation du pesticide dont ils sont issus et, dans certains cas, avoir un impact écologique plus large comme c'est le cas pour les anilines et les phénols chlorosubstitués (BARTHA, 1968 ; HUBER et al., 1980). Le 2,4-dichlorophénol, par exemple, est un intermédiaire de la dégradation du 2,4-D dans le sol (FosTER \& MC KERCHER, 1973 ; KUNC \& RYBAROVA, 1983 ; SMITH, 1985). Bien qu'il s'agisse aussi d'une substance à activité hormonale (ANDO et al., 1970), ou antibactérienne (SIUDA \& DE BERNADIS, 1973) naturellement produite par certains micro-organismes, dont un Penicillium $s p$., on peut supposer que son apparition à partir de 2,4-D peut se produire en quantités dont l'impact écotoxicologique dépasse largement le rôle qui est naturellement le sien dans le cadre des relations entre organismes du sol. Dans ce travail nous avons cherché à vérifier que le 2,4-dichlorophénol était un intermédiaire de la dégradation du 2,4-D dans un sol où nous avions par ailleurs étudié la cinétique de sa dégradation (FOURNIER et al., 1981 ; Soulas et al., 1983). Pour cela, afin d'éviter de mettre en œuvre une technique analytique longue et aléatoire (NARANG et al., 1983) nous avons préféré utiliser une méthode cinétique consistant à modifier la courbe de dégradation d'un composé par adjonction de différentes quantités soit de l'un de ses précurseurs dans la séquence métabolique de sa dégradation, soit au contraire de l'un de ses produits de transformation. Les conséquences de ces interrelations cinétiques sur l'activité ainsi que l'importance de la microflore dégradant le 2,4-D ont aussi été étudiées.

\section{MATÉRIEL ET MÉTHODE}

\section{A. Préparation, traitement du sol, dispositif expé- rimental}

Nous avons utilisé un sol limono-argileux dont les principales caractéristiques sont données dans le tableau 1. Le sol a d'abord été soumis à un séchage modéré jusqu'à une humidité correspondant à $60 \%$ de l'humidité équivalente et compatible avec un tamisage. Seule la fraction de sol comprise entre 2 et $3 \mathrm{~mm}$ est conservée puis répartie dans des fioles de verre de $250 \mathrm{ml}$ à raison de l'équivalent de $50 \mathrm{~g}$ de sol sec par fiole. Chaque échantillon a reçu un volume d'eau nécessaire pour ajuster son humidité à $70 \%$ de l'humidité équivalente puis $1 \mathrm{ml}$ d'une solution dans du tampon phosphate $(\mathrm{pH}=7 ; \mathrm{M} / 20)$ de $2,4-\mathrm{D}$, de 2,4dichlorophénol $(2,4-\mathrm{DCP})$ ou d'un mélange des deux. La teneur finale en eau du sol était alors égale à $78 \%$ de l'humidité équivalente. Pour suivre la dégradation d'un produit, des traces de ce produit radioactif ont été ajoutées à la solution du produit froid correspondant.
L'échantillon de sol ainsi traité a été placé dans un bocal en verre de 2 I hermétique. Ce bocal contient en outre $10 \mathrm{ml}$ d'eau, pour maintenir l'humidité de l'échantillon de sol, et une fiole de scintillation de $20 \mathrm{ml}$ contenant $10 \mathrm{ml}$ de $\mathrm{NaOH}, 0,2 \mathrm{~N}$. Celle-ci était destinée à piéger le ${ }^{14} \mathrm{CO}_{2}$ dégagé au cours de la dégradation dont la progression était suivie par remplacement périodique des pièges et analyse par scintillation liquide de la radioactivité qu'ils contiennent sous forme de carbonates. Dans toutes les expériences, l'incubation des échantillons se fait en salle à température contrôlée et fixée à $20^{\circ} \mathrm{C} \pm 0,5^{\circ} \mathrm{C}$.

\section{B. Plans expérimentaux}

1. Première expérience. Comparaison des cinétiques de dégradation du 2,4-D et du 2,4-DCP. Adaptation du sol

Nous avons comparé les cinétiques de dégradation du 2,4-D et du 2,4-DCP à deux concentrations différentes et étudié l'effet de ces différents traitements sur l'adaptation de la microflore du sol à la dégradation du 2,4-D. Pour cela, deux séries d'échantillons ont été préparées (tabl. 2). Dans la première (traitements $a$ à d), les échantillons de sol ont reçu $1 \mathrm{ml}$ d'une solution radioactive de l'un des deux produits étudiés, chacun à deux doses d'application. Les produits sont uniformément marqués sur le cycle. Les activités spécifiques de ces solutions étaient respectivement de $0,3 \mu \mathrm{Ci} \mathrm{ml}{ }^{-1}$ pour le $2,4-\mathrm{D}$ et de $0,1 \mu \mathrm{Ci} \mathrm{ml}{ }^{-1}$ pour le 2,4-DCP ; elles étaient indépendantes de la concentration des produits. Les doses d'application, identiques pour les deux produits, étaient respectivement de $1,5 \cdot 10^{-3}$ minole (produit) $\mathrm{kg}^{-1}$ (sol) pour la faible dose (D1), représentative de la dose normalement utilisée en agriculture, et de $1,5 \cdot 10^{-1}$ mmole $\mathrm{kg}^{-1}$ pour la dose élevée (D2). Chaque traitement a été répété quatre fois. Ces échantillons nous ont servi à comparer les cinétiques de dégradation du 2,4-D ou du 2,4-DCP, aux deux concentrations retenues, en suivant le dégagement de ${ }^{14} \mathrm{CO}_{2}$ pendant 86 jours.

Dans la seconde série destinée à apprécier un éventuel effet d'adaptation du sol à la dégradation du 2,4-D, les échantillons ont reçu les mêmes traitements à la seule différence près que les solutions des différents produits ne contenaient pas de radioactivité (traitements $e$ à $h$ ). En outre, 8 échantillons ont reçu uniquement du tampon (traitement $i$ ). Après 86 jours d'incubation ces échantillons ont reçu un second traitement par $1 \mathrm{ml}$ de solution de $2,4-\mathrm{D}$ radioactive (activité spécifique : $0,1 \mu \mathrm{Ci} \mathrm{ml} \mathrm{m}^{-1}$, quelle que soit la dose) et apportant, pour un échantillon donné, une quantité de $2,4-\mathrm{D}$ égale à la quantité de produit $(2,4-\mathrm{D}$ ou 2,4 -

TABLEAU I

Caractéristiques physico-chimiques du sol utilisé.

Physico-chemical characteristics of the soil.

\begin{tabular}{|c|c|c|c|c|c|c|}
\hline$\underset{0 \%}{\text { Argile }}$ & $\underset{\%}{\operatorname{Limon}}$ & $\begin{array}{c}\text { Sable } \\
\% 0\end{array}$ & $\begin{array}{c}\text { CEC } \\
\mathrm{meq} / 100 \mathrm{~g}\end{array}$ & $\underset{\%}{\text { M.O. }}$ & $\begin{array}{c}\mathrm{pH} \\
\text { (eau) }\end{array}$ & $\begin{array}{c}\text { Humidité } \\
\text { équivalente }(\%)\end{array}$ \\
\hline 32,7 & 54,0 & 13,4 & 19,8 & 2,1 & 7,4 & 23,4 \\
\hline
\end{tabular}


TABLEAU 2

Première expérience : plan expérimental.

First experiment : experimental scheme.

\begin{tabular}{|c|c|c|c|c|c|c|}
\hline \multirow[b]{2}{*}{ Produit } & \multicolumn{3}{|c|}{ TRAITEMENT $1(\mathrm{t}=0 \mathrm{j})$} & \multicolumn{3}{|c|}{ TRAITEMENT $2(\mathrm{t}=86 \mathrm{j})$} \\
\hline & Dose & $\begin{array}{l}\text { Nombre de } \\
\text { répétitions }\end{array}$ & $\begin{array}{l}\text { Nom du } \\
\text { traitement }\end{array}$ & Produit & Dose & $\begin{array}{l}\text { Nombre de } \\
\text { répétitions }\end{array}$ \\
\hline $\begin{array}{c}{ }^{14} \mathrm{C}-2,4 \mathrm{D} \\
(0,3 \mu \mathrm{Ci})\end{array}$ & D1 & 4 & a & & & \\
\hline$-i d-$ & $\mathrm{D} 2(=100 \mathrm{D} 1)$ & 4 & $b$ & & & \\
\hline \multirow{2}{*}{$\begin{array}{c}{ }^{14} \mathrm{C}-2,4-\mathrm{DCP} \\
(0,1 \mu \mathrm{Ci}) \\
-i d-\end{array}$} & D1 & 4 & c & & & \\
\hline & D2 & 4 & $\mathrm{~d}$ & & & \\
\hline $2,4-\mathrm{D}$ & D1 & 4 & $\mathrm{e}$ & $\begin{array}{c}{ }^{14} \mathrm{C}-2,4-\mathrm{D} \\
(0,1 \mu \mathrm{Ci})\end{array}$ & D1 & 4 \\
\hline$-i d-$ & D2 & 4 & $\mathrm{f}$ & $-i d-$ & $\mathrm{D} 2$ & 4 \\
\hline 2,4-DCP & D1 & 4 & $\mathrm{~g}$ & $-i d-$ & D1 & 4 \\
\hline$-i d-$ & $\mathrm{D} 2$ & 4 & $\mathrm{~h}$ & $-i d-$ & D2 & 4 \\
\hline \multirow[b]{2}{*}{ Tampon seul } & & 4 & $\mathrm{i}$ & $-i d-$ & D1 & 4 \\
\hline & - & 4 & $\mathrm{i}$ & $-i d-$ & D2 & 4 \\
\hline
\end{tabular}

DCP) introduite lors du premier traitement. Les échantillons traités avec du tampon ont été séparés en 2 lots de 4 , chaque lot recevant une des deux solutions radioactives de $2,4-\mathrm{D}$.

\section{Deuxième expérience. Cinétique de la dégradation} du 2,4-D en présence de 2,4-DCP

Elle avait pour but d'apprécier l'effet du 2,4-DCP sur la cinétique de dégradation du 2,4-D dans le sol. Ainsi que l'indique le tableau 3 , nous avons suivi simultanément 2 séries d'échantillons regroupant cha-

TABLEAU 3

Deuxième expérience : plan expérimental (ce plan vaut pour le 2,4-D marqué sur la chaîne et sur le cycle).

Second experiment : experimental scheme

(this scheme holds for chain- or ring-labelled 2,4-D).

\begin{tabular}{|c|c|}
\hline $\begin{array}{c}{ }^{14} \mathrm{C}-2,4-\mathrm{D} \\
\text { concentration } \\
\left(10^{-3} \text { mmole } \mathrm{kg}^{-1}\right)\end{array}$ & $\begin{array}{c}\text { 2,4-DCP } \\
\text { concentration } \\
\left(10^{-3} \text { mmole } \mathrm{kg}^{-1}\right)\end{array}$ \\
\hline 1,5 & $\begin{array}{r}0 \\
1,5 \\
6,0 \\
24,0 \\
96,0\end{array}$ \\
\hline 150 & $\begin{array}{r}0 \\
1,5 \\
6,0 \\
24,0 \\
96,0\end{array}$ \\
\hline
\end{tabular}

cune les mêmes traitements, la seule différence entre elles résidant dans l'utilisation de 2,4-D radioactif marqué soit sur la chaîne, soit sur le cycle afin de suivre différentes étapes de la dégradation. L'activité spécifique de toutes les solutions de traitement était uniforme et égale à $0,1 \mu \mathrm{Ci}(2,4-\mathrm{D}) \mathrm{ml}^{-1}$. Dans chaque série nous avons étudié la dégradation du 2,4-D à deux concentrations initiales : $1,5 \quad 10^{-3}$ mmole $\mathrm{kg}^{-1}$ et

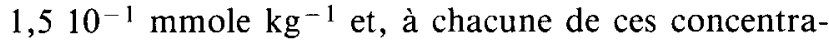
tions, à cinq concentrations en 2,4-DCP : $0,1,510^{-3}$, $6,010^{-3}, 2,410^{-2}$ et $9,610^{-2}$ mmole $\mathrm{kg}^{-1}$. Chaque traitement a été répété 4 fois.

Lorsque la dégradation a été considérée comme achevée soit après 101 jours à la faible dose et 138 jours à la forte dose, nous avons déterminé la radioactivité incorporée dans la biomasse par une technique de fumigation au chloroforme (KASSIM et al., 1981 ; STOTT et al., 1983 ; SOULAS et al., 1984). Les échantillons de sol ont été soumis à des vapeurs de chloroforme pendant 16 heures. Après élimination complète des vapeurs résiduelles de chloroforme par plusieurs mises sous vide successives, les échantillons ont été remis en incubation. Le dégagement de ${ }^{14} \mathrm{CO}_{2}$ a été mesuré après 7,14 et 28 jours. La part du ${ }^{14} \mathrm{CO}_{2}$ venant de la biomasse tuée par le chloroforme a été estimée en soustrayant de la quantité de ${ }^{14} \mathrm{CO}_{2}$ dégagée pendant les 14 premiers jours celle qui s'est dégagée pendant les 14 jours suivants (CHAUSSOD \& NICOLARDOT, 1982).

\section{Troisième expérience. Cinétique de la dégradation du 2,4-DCP en présence de 2,4 D}

Elle visait à apprécier l'effet de différentes concentrations de 2,4-D sur la dégradation du 2,4-DCP dans le sol. Ainsi, tous les échantillons de sol ont reçu la même quantité de 2,4-DCP radioactif $(0,1 \mu \mathrm{Ci}$ par échantillon) et de 2,4-DCP froid : sa concentration

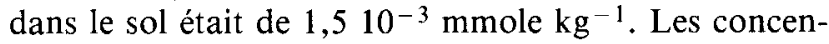
trations en 2,4-D des solutions de traitement étaient par contre différentes. On a ainsi préparé 6 lots de 4 échantillons dans lesquels les concentrations en 2,4-D étaient respectivement de $0,3,010^{-3}, 6,010^{-3}$,

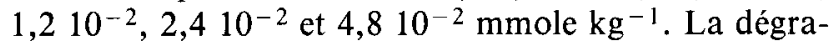
dation du 2,4-DCP a été suivie par mesure du ${ }^{14} \mathrm{CO}_{2}$ dégagé. 


\section{RÉSULTATS}

\section{A. Comparaison des cinétiques de dégradation du 2,4-D et du 2,4-DCP. Adaptation du sol}

La comparaison des figures $1 a$ et $b$ permet de voir l'effet de la dose sur les cinétiques de dégradation respectives du 2,4-DCP et du 2,4-D. Pour le 2,4-DCP l'effet de la dose est relativement peu marqué puisque les pourcentages de dégradation en fin d'expérience atteignent respectivement $32,5 \%$ à la dose D1 et $24,7 \%$ à la dose D2. Pour le 2,4-D l'effet de la dose se manifeste de façon beaucoup plus nette. En particulier
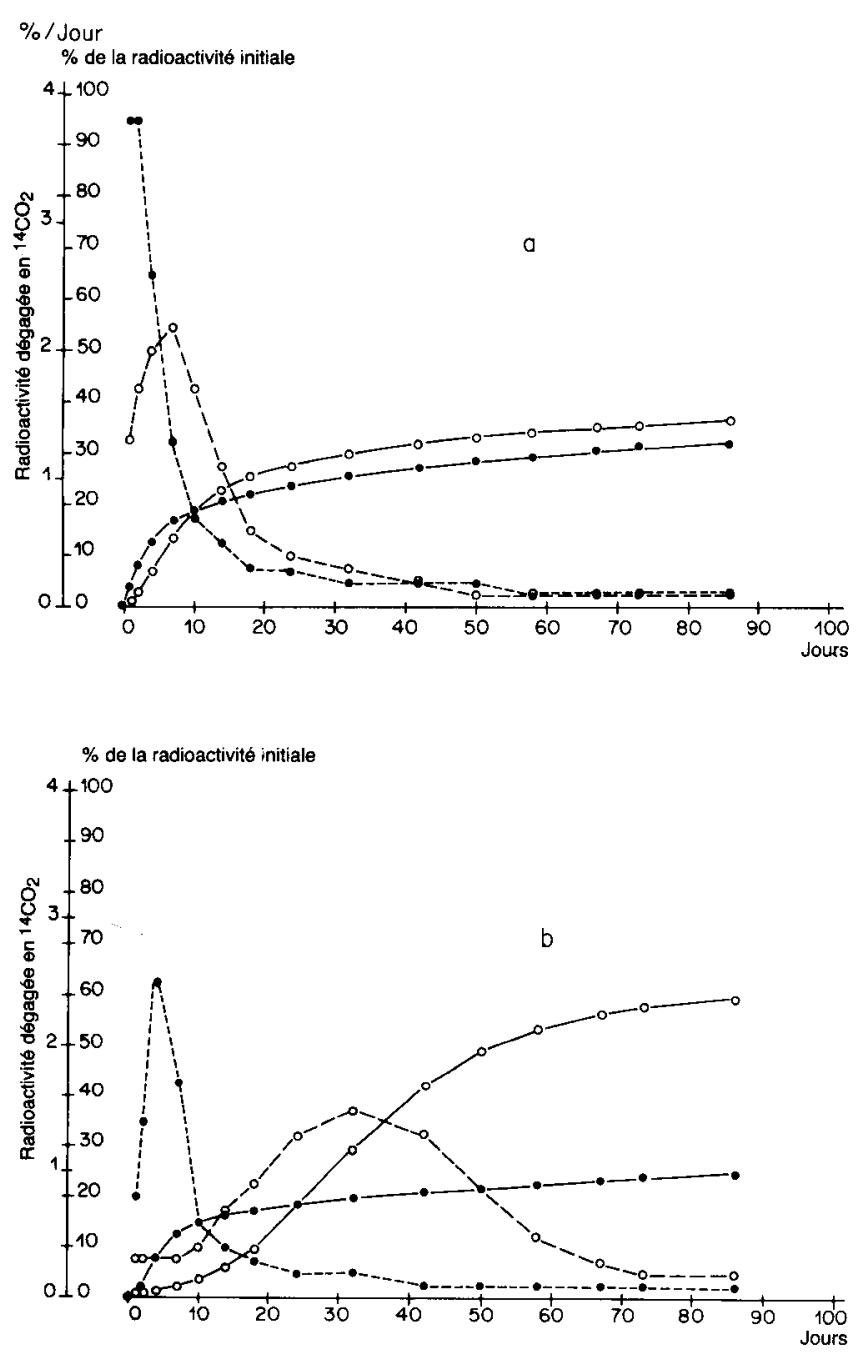

Figure 1

Comparaison des cinétiques de dégradation du 2,4-D et du 2,4dichlorophénol dans le sol:

- Q:2,4-D $\bullet: 2,4-$ dichlorophénol,

- trait plein : dégradation cumulée exprimée en \% de la radioactivité initiale ajoutée; trait interrompu: vitesse de dégradation exprimée en p. 100 par jour de la radioactivité initiale,

- a : $1,510^{-3}$ mmole (produit) $\mathrm{kg}^{-1}$ (sol) ; b : $1,510^{-1}$ mmole (produit) $\mathrm{kg}^{-1}$ (sol).

(Chaque point correspond à la moyenne de 4 répétitions.)

Comparison between breakdown kinetics of 2,4-D and 2,4dichlorophenol in the soil :

- $\bigcirc: 2,4-D ; \bullet: 2,4$-dichlorophenol,

- solid line: cumulated degradation expressed as $\%$ of initially added radioactivity; dashed line : degradation rate expressed as $\%$ per day of initial radioactivity,

- a : $1.510^{-3}$ mmole (chemical) $\mathrm{kg}^{-1}$ (soil) ; b : $1.510^{-1}$ mmole (chemical) $\mathrm{kg}^{-1}$ (soil).

(Each point is the mean of 4 replicates.) une augmentation de la dose non seulement augmente le pourcentage de dégradation $(60 \%$ à la dose D2 contre $36,7 \%$ à la dose D1), mais modifie également la cinétique de dégradation; la dose la plus élevée fait apparaître une phase de latence de 7 jours au-delà de laquelle on constate une augmentation de la vitesse de dégradation. Les courbes de dégradation journalière confirment la rapide stabilisation de la vitesse de dégradation du 2,4-DCP qui devient très faible après 10 jours d'expérience.

Sur les figures $2 a$ et $b$ sont représentées les courbes de dégradation du 2,4-D appliqué sur des sols ayant reçu 86 jours auparavant soit du tampon (témoin), soit du 2,4-D, soit du 2,4-DCP. On observe l'effet positif déjà connu d'un premier apport 2,4-D sur la dégradation d'un second apport. Mais il s'avère également que le 2,4-DCP à la dose la plus élevée favorise la dégradation d'un apport ultérieur de 2,4-D.
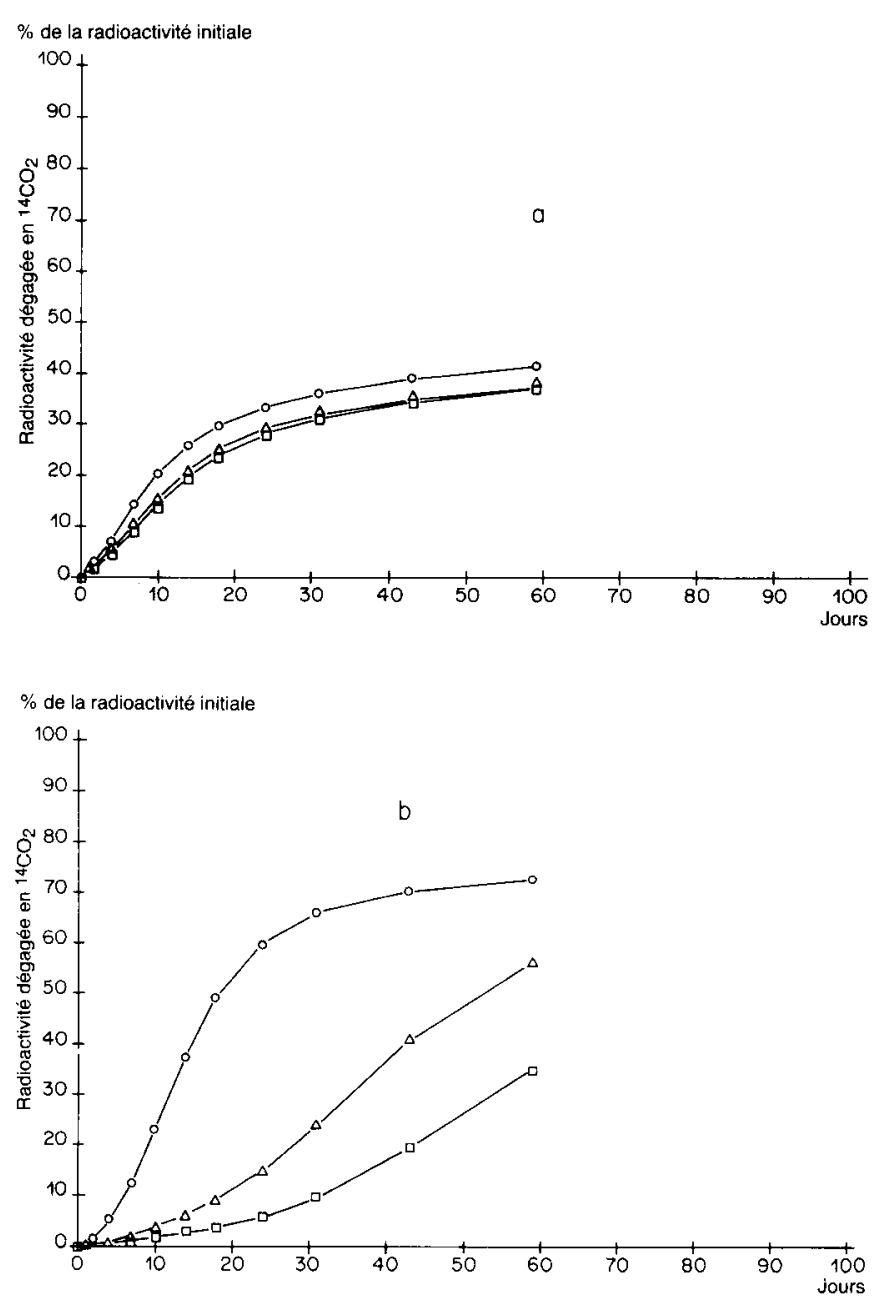

Figure 2

Modifications de la cinétique de dégradation du 2,4-D induites par différents traitements préalables du sol:

- $\square$ : sol témoin; $\triangle$ : sol ayant reçu du 2,4-dichlorophénol; $O$ : sol ayant reçu du $2,4-D$,

- a : 1,510 $10^{-3}$ mmole $(2,4-\mathrm{D}) \mathrm{kg}^{-1}$ (sol) ; b : $1,510^{-1}$ mmole (2,4D) $\mathrm{kg}^{-1}$ (sol).

(Chaque point correspond à la moyenne de 4 répétitions.)

Modification of the breakdown kinetics of 2,4-D after different preliminary soil treatments :

- $\square:$ control; $\triangle:$ soil previously treated with 2,4dichlorophenol; 0 : soil previously treated with $2,4-D$,

- a : $1.510^{-3}$ mmole $(2,4-D) k^{-1}$ (soil) ; b : $1.510^{-1}$ mmole (2,4D) $\mathrm{kg}^{-i}$ (soil). 


\section{B. Cinétique de la dégradation du 2,4-D en présence de 2,4-DCP}

La figure 3 indique clairement que des concentrations en 2,4-DCP variant de $1,510^{-3}$ à $9610^{-3}$ mmole $\mathrm{kg}^{-1}$ (soit dans un rapport de 1 à 64) n'ont pas d'influence significative sur le pourcentage de dégradation du 2,4-D quelle que soit l'étape de dégradation considérée, repérée par la position du marquage sur la molécule. Cependant, à la dose la plus faible en herbicide et pour ce qui concerne le produit marqué sur la chaîne (fig. $3 a$ ) on observe après 11 jours d'incubation une inhibition significative (PPDS à 5 p. $100=2,2$ p. 100) mais temporaire, du dégagement de ${ }^{14} \mathrm{CO}_{2}$ et ce, uniquement à la dose la plus élevée en 2,4-DCP. L'incorporation correspondante de radioactivité dans la biomasse $(5,20 \mathrm{p} .100)$ est statistiquement plus faible (au seuil de 5 p. 100) que dans les autres traitements (tabl. 4). Avec le 2,4-D marqué sur le cycle, seuls les deux traitements correspondant aux doses les plus élevées en 2,4-DCP ont des effets significatifs (PPDS à
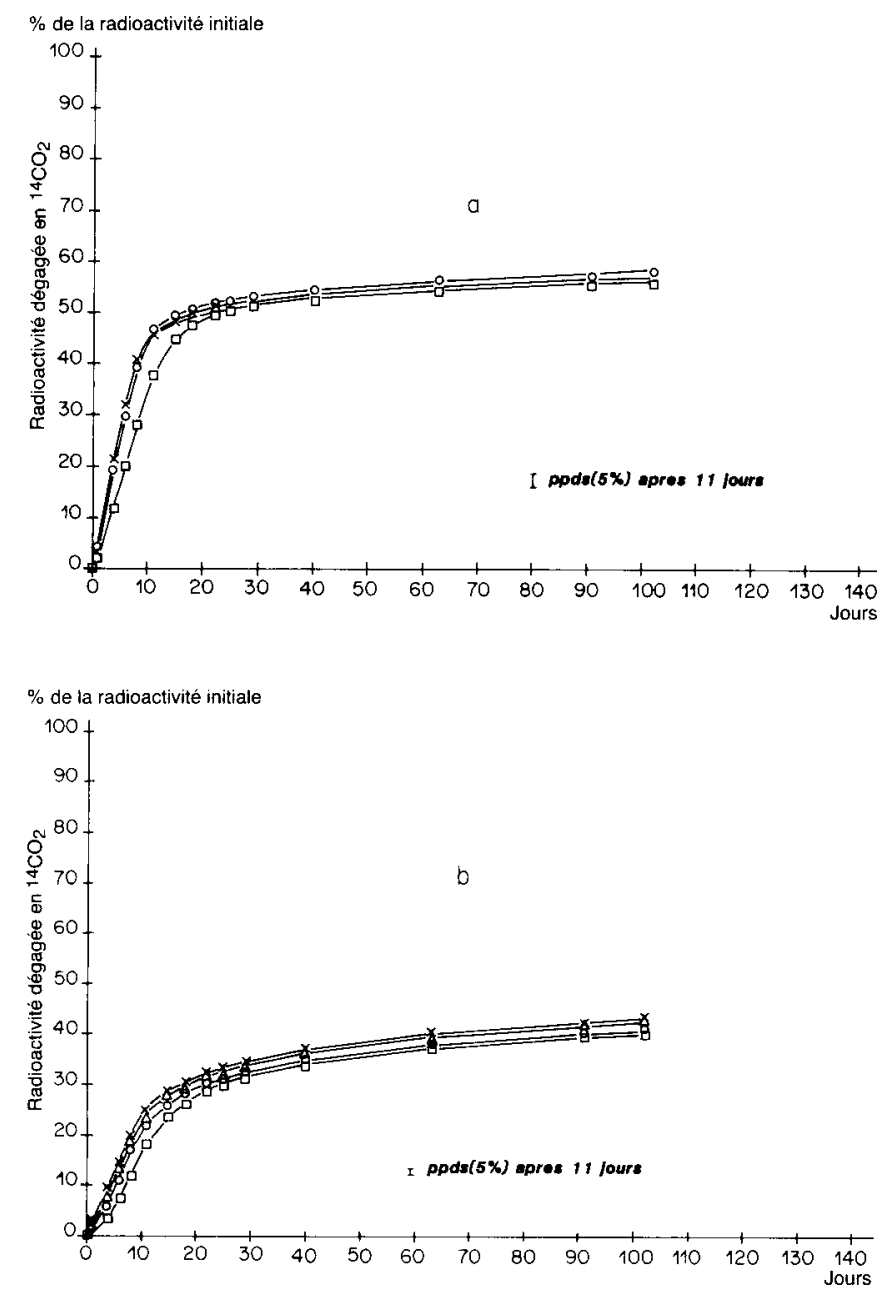

Figure 3

Cinétiques de dégradation du 2,4-D en présence de 2,4-dichlorophénol.

- a : $1,510^{-3}$ mmole $\mathrm{kg}^{-1}$, 2,4-D marqué sur la chaîne; - b: 1,5 $10^{-3}$ mmole $\mathrm{kg}^{-1}, 2,4-\mathrm{D}$ marqué sur le cycle; - c : 1,5 $10^{-1}$ mmole $\mathrm{kg}^{-1}, 2,4-D$ marqué sur la chaîne; - d : 1,5 $10^{-1}$ mmole $\mathrm{kg}^{-1}, 2,4-\mathrm{D}$ marqué sur le cycle. - Concentrations en 2,4-dichlorophénol : $\square: 9610^{-3}$ mmole $\mathrm{kg}^{-1} ; 0: 2410^{-3}$ mmole $\mathrm{kg}^{-1} ; \triangle: 610^{-3}$ mmole $\mathrm{kg}^{-1} ; \bullet$ : I,5 $10^{-3}$ mmole $\mathrm{kg}^{-1}: \times:$ témoin.

(Chaque point correspond à la moyenne de 4 répétitions.)
5 p. $100=1,5$ p. 100) tant sur la cinétique d'évolution du ${ }^{14} \mathrm{CO}_{2}$ après 11 jours que sur l'incorporation de radioactivité dans la biomasse (fig. $3 b$; tabl. 4).

Pour de fortes doses de 2,4-D (fig. $3 c$ et $d$ ) seules les deux doses de phénol les plus élevées réduisent significativement la quantité de ${ }^{14} \mathrm{CO}_{2}$ dégagée après 63 jours à partir de 2,4-D marqué sur la chaîne (PPDS à $5 \mathrm{p} .100=3 \mathrm{p} .100$ ). Aucun effet significatif n'apparaît avec du 2,4-D marqué sur le cycle (PPDS à 5 p. $100=10,5$ p. 100 ). Enfin, pour une position donnée du marquage, la radioactivité incorporée dans la biomasse ne change pas quelle que soit la concentration en 2,4-DCP.

\section{Cinétique de la dégradation du 2,4-DCP en pré- sence de 2,4-D}

La figure 4 montre que le 2,4-D, quelle que soit sa concentration, contribue de façon significative (PPDS à 5 p. 100 après 17 jours $=0,5$ p. 100) à diminuer la
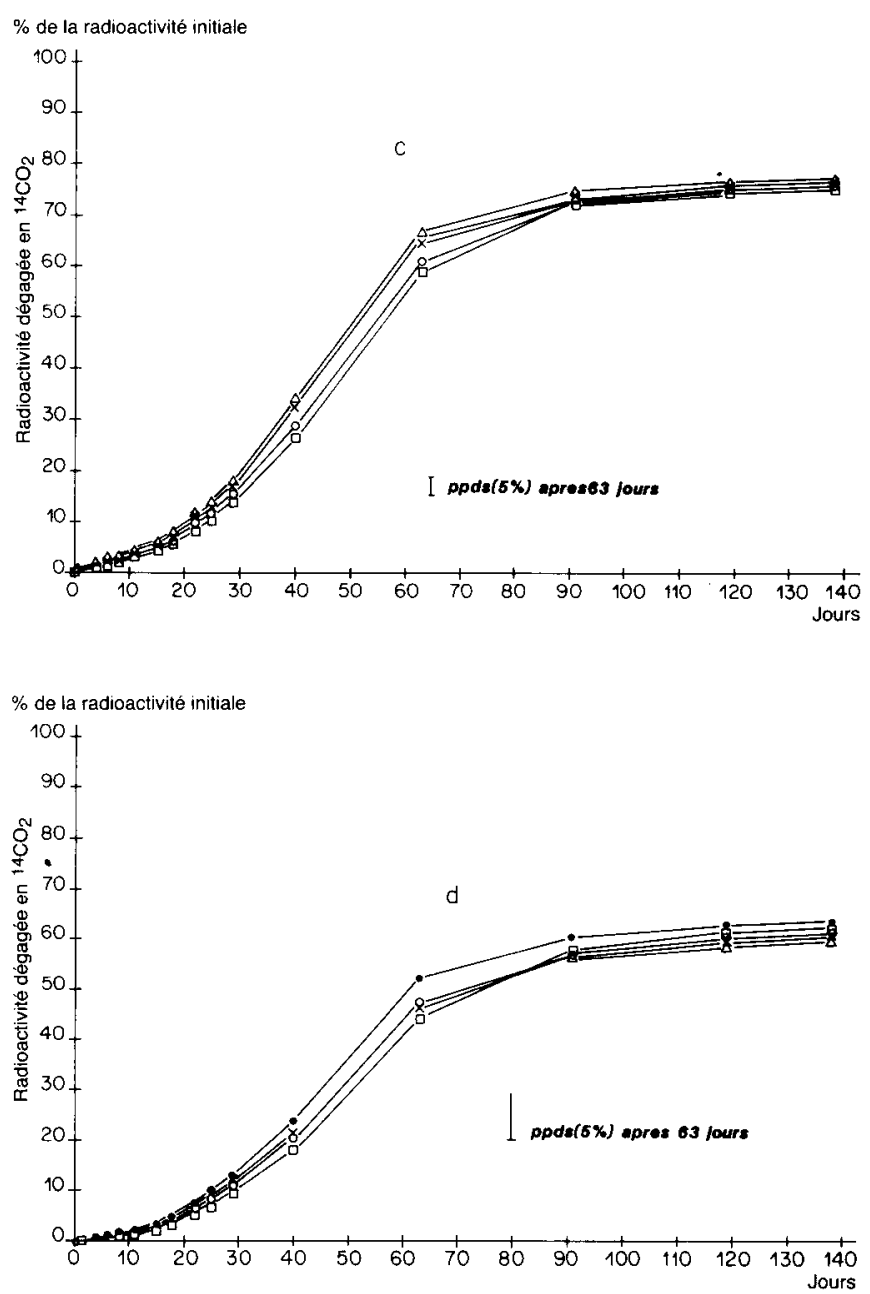

Breakdown kinetics of 2,4-D with 2,4-dichlorophenol.

- a : $\quad 1.510^{-3}$ mmole $\mathrm{kg}^{-1}$, chain-labelled 2,4-D - b : $1.510^{-3}$ mmole $\mathrm{kg}^{-1}$, ring-labelled 2,4-D ; - c: $1.510^{-1}$ mmole $\mathrm{kg}^{-1}$, chain-labelled $2,4-D ;-\mathrm{d}: 1.510^{-1}$ mmole $\mathrm{kg}^{-1}$, ring-labelled 2,4-D.

- 2,4-dichlorophenol concentration : $\square: 9610^{-3}$ mmole $\mathrm{kg}^{-1}$; $\mathrm{O}: 2410^{-3}$ mmole $\mathrm{kg}^{-1} ; \triangle: 610^{-3}$ mmole $\mathrm{kg}^{-1} ; \bullet: 1.510^{-3}$ mmole $\mathrm{kg}^{-1} ; \times$ : control.

(Each point is the mean of 4 replicates.) 
TABLEAU 4

Dégagement corrigé de ${ }^{14} \mathrm{CO}_{2}$ après fumigation exprimé en \% de la radioactivité initiale. $f^{*}$ : traitement significativement différent du témoin.)

Corrected ${ }^{14} \mathrm{CO}_{2}$ release after fumigation expressed as $\%$ of initially added radioactivity. ${ }^{*}$ : statistically different treatment.)

\begin{tabular}{|c|c|c|c|c|}
\hline \multirow{2}{*}{$\begin{array}{c}\text { Concentration en } \\
2,4-\mathrm{DCP} \\
\left(10^{3} \text { mmole } \mathrm{kg}^{-1}\right)\end{array}$} & \multicolumn{4}{|c|}{ Concentration en 2,4-D } \\
\hline & ${ }^{14} \mathrm{C}$-Chaîne & ${ }^{14} \mathrm{C}-\mathrm{Noyau}$ & ${ }^{14} \mathrm{C}$-Chaîne & ${ }^{14} \mathrm{C}-$ Noyau \\
\hline 96,0 & $5,20^{*}$ & $2,43^{*}$ & 1,48 & 1,25 \\
\hline 24,0 & 5,60 & $2,68^{*}$ & 1,55 & 1,13 \\
\hline 6,0 & 5,53 & 2,83 & 1,50 & 1,15 \\
\hline 1,5 & 5,70 & 2,90 & 1,50 & 1,08 \\
\hline 0 & 5,60 & 2,95 & 1,58 & 1,15 \\
\hline $\operatorname{PPDS}(5 \%)$ & 0,31 & 0,20 & 0,18 & 0,17 \\
\hline
\end{tabular}

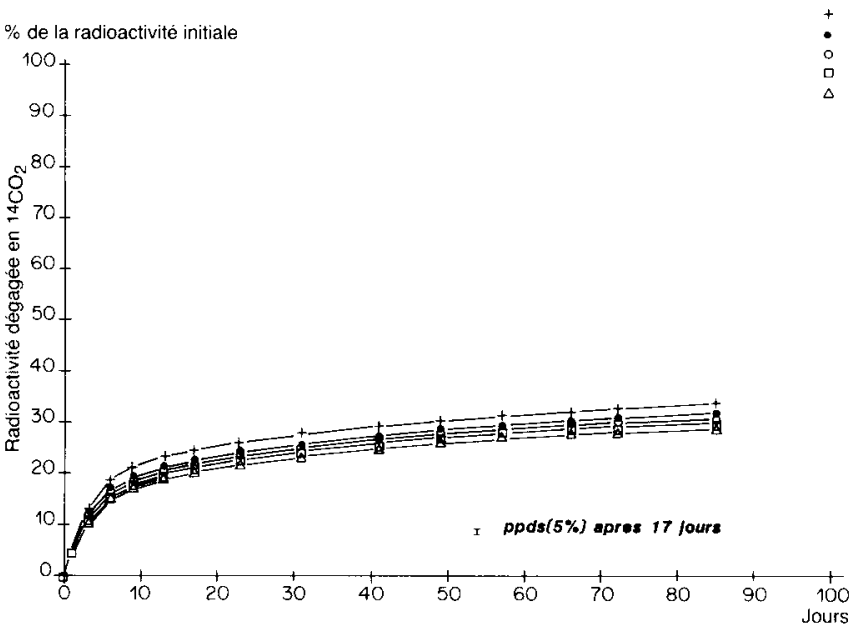

Figure 4

Cinétique de la dégradation du 2,4-dichlorophénol en présence de 2,4-D.

Concentration en 2,4-D en mmole $\mathrm{kg}^{-1}: \square: 4,810^{-2} ; 0$ : $2,410^{-2} ; \triangle: 1,210^{-2} ; \times: 6,010^{-3} ; \bullet: 3,010^{-3} ;+$ : témoin. (Chaque point correspond à la moyenne de 4 répétitions.)

Breakdown kinetics of 2,4-dichlorophenol with 2,4-D.

2,4-D concentration in mmole $\mathrm{kg}^{-1}: \square: 4.8 \quad 10^{-2} ; 0$ : $2.410^{-2} ; \triangle: 1.210^{-2} ; \times: 6.010^{-3} ;: 3.010^{-3} ;+$ : control. (Each point is the mean of 4 replicates.)

quantité de radioactivité dégagée sous forme ${ }^{14} \mathrm{CO}_{2}$ à partir de ${ }^{14} \mathrm{C}-2,4-\mathrm{DCP}$. Aucune différence significative n'apparaît cependant entre traitements correspondant à différentes doses de 2,4-D.

\section{DISCUSSION}

On peut penser que les formes différentes qui caractérisent les courbes de dégradation du 2,4-D et du 2,4DCP, surtout à la concentration relativement élevée, sont l'indication d'une différence de comportement des microflores dégradant ces deux composés. Dans le cas du 2,4-D, on sait que, suivant la concentration, ce ne sont pas les mêmes micro-organismes qui jouent le rôle essentiel dans la dégradation (FouRNIER et al., 1981). En particulier, une augmentation de la concentration en 2,4-D renforce le rôle de micro-organismes relativement spécifiques mais initialement peu nom- breux. En effet, une forte concentration permet une croissance de ces micro-organismes et se traduit par une augmentation progressive de la vitesse de dégradation. Nos résultats confirment une fois de plus cette explication. En effet, la vitesse de dégradation du 2,4-D augmente immédiatement après son apport à faible dose mais seulement après 7 jours à forte dose. Ceci signifie que la microflore dégradant spécifiquement le 2,4-D est naturellement présente dans le sol à un niveau de population qui lui permet à faible dose d'exprimer immédiatement son activité dégradante. A dose plus élevée, un réajustement de ce niveau serait nécessaire dont les effets sur la vitesse de dégradation du 2,4-D ne se feraient sentir qu'après une semaine d'incubation. En d'autres termes, la microflore dégradante spécifique initialement présente dans le sol serait un facteur limitant de la vitesse de dégradation.

Les choses se présentent différemment avec le 2,4-DCP. En effet, alors que les concentrations en 2,4-DCP varient dans un rapport de 1 à 100, la forme des courbes de dégradation est peu modifiée et les vitesses de dégradation, exprimées en pourcentages, sont relativement comparables excepté pendant une période initiale de 4 jours. Ceci suggère fortement que c'est surtout la concentration en phénol qui limite la vitesse de dégradation. Dans une étude récente CHENG et al. (1983) comparent les courbes de dégradation du 2,4-DCP à deux concentrations ( 10 et $\left.100 \mathrm{mg} \mathrm{kg}^{-1}\right)$ et observent entre elles des différences identiques à celles que nous observons dans notre étude pour le 2,4-D. Ceci tendrait à montrer qu'à des concentrations en 2,4-DCP supérieures à celles auxquelles nous avons nous-mêmes travaillé, une croissance microbienne deviendrait possible. Ceci serait en accord avec l'hypothèse de BAKER \& MAYFIELD (1980) et BAKER et al. (1980) qui supposent que la microtlore du sol dégradant le 2,4-DCP serait relativement abondante comparée à celle qui dégrade le 2,4-D. Il ne serait donc pas étonnant dans ces conditions que le seuil de concentration au-delà duquel une croissance se manifeste soit plus élevé avec le 2,4-DCP qu'avec le 2,4-D.

En outre, le chlorophénol semblerait être rapidement stabilisé dans le sol (CHENG et al., 1983) soit parce qu'il participe à des réactions de polymérisation après autooxydation (HAIDER et al., 1975 ; MARTIN et al., 1979) ou par action enzymatique (BollaG et al., 1980 ; MiNARD et al., 1981) ou encore parce qu'il se lierait à certains constituants organiques du sol (STE- 
VENSON, 1972). La diminution immédiate et prononcée, surtout à faible concentration, de la vitesse de dégradation de ce composé que nous observons dans nos expériences alors que subsiste encore dans le sol un pourcentage important de radioactivité semblerait être en accord avec une rapide stabilisation.

L'effet d'adaptation à la dégradation du 2,4-D que nous observons après un traitement au 2,4-DCP n'est pas une preuve directe du fait que ce composé serait un intermédiaire de la dégradation du 2,4-D. Il est cependant évident qu'une fraction non négligeable de la population microbienne dégradant le phénol chloré dégrade aussi le 2,4-D. Les résultats des études cinétiques ne permettent pas non plus de conclure quant à l'appartenance de ces deux composés à une même voie métabolique. L'effet de stabilisation dont il est question précédemment permettrait cependant d'interpréter les résultats observés. En effet, si le produit de dégradation du 2,4-D est bien du 2,4-DCP celui-ci apparaît dans la biomasse dégradante où il est soustrait aux effets stabilisateurs du milieu environnant. Ce n'est pas le cas du phénol exogène. Ainsi, suivant qu'il est ajouté directement au sol ou sous la forme d'un « précurseur » tel que le 2,4-D, le 2,4-DCP évoluerait dans des « compartiments » différents entre lesquels les échanges pourraient être relativement limités. Cette dernière constatation aurait des conséquences importantes sur le mode d'appréciation de l'impact sur la microflore du sol, d'un intermédiaire de dégradation tel que le 2,4-DCP, dont l'activité biologique est limitée en raison d'une interaction avec le milieu physique environnant. Au contraire, cette bioactivité pourrait s'exprimer plus facilement lorsque de tels composés sont produits et s'accumulent dans la biomasse à partir d'un précurseur plus mobile dans le sol tel que le 2,4-D, qui pourrait éventuellement ne pas manifester lui-même de toxicité importante à l'égard de la microflore du sol.

\section{CONCLUSION}

Les résultats rapportés ne nous ont pas permis de conclure quant aux interrelations cinétiques de la dégradation du 2,4-D et du 2,4-dichlorophénol dans le sol. La relative indépendance d'évolution dans le sol que nous avons observée entre les deux produits nous a cependant permis de mieux comprendre certaines caractéristiques de leur évolution respective. Au plan biologique tout d'abord, ajoutés directement au sol, ces produits subiraient l'action de deux microflores de tailles très différentes. Au plan physico-chimique ensuite, le 2,4-D est connu pour être peu fixé dans le sol et reste en conséquence relativement accessible aux micro-organismes ; il n'en est pas de même avec le 2,4dichlorophénol qui, au contraire, semble être stabilisé et inactivé très rapidement après son introduction dans le sol, l'empêchant ainsi de perturber l'évolution intracellulaire du 2,4-dichlorophénol d'origine métabolique.

Reçu le 19 avril 1985 Accepté le 10 décembre 1986.

\section{RÉFÉRENCES BIBLIOGRAPHIQUES}

Ando K., Kato A., Suzuki S., 1970. Isolation of 2,4-dichlorophenol from a soil fungus and its biological significance. Biochem. biophys. Res. Commun., 39 (6), 1104-1107.

Baker M. D., Mayfield C. I., 1980. Microbial and non-biological decomposition of chlorophenols and phenol in soil. Water Air Soil Pollut., 13, 411-424.

Baker M. D., Mayfield C. I., Innis W. E., 1980. Degradation of chlorophenols in soil, sediment and water at low temperature. Water Res., 14, 1765-1771.

Bartha R., 1968. Biochemical transformations of anilide herbicides in soil. J. agric. Food Chem., 16 (4), 602-604.

Bollag J. M., Liu S. Y., Minard R. D., 1980. Cross-coupling of phenolic humus constituents and 2,4-dichlorophenol. Soil Sci. Soc. Am. $J ., 44,52-56$.

Chaussod R., Nicolardot B., 1982. Mesure de la biomasse microbienne dans les sols cultivés. I. - Approche cinétique et estimation simplifiée du carbone facilement minéralisable. Rev. Ecol. Biol. Sol, $19(4), 501-512$.

Cheng H. H., Haider K., Harper S. S., 1983. Catechol et chlorocatechols in soil : degradation and extractability. Soil Biol. Biochem., $15(3), 311-317$.

Foster R. K., Mc Kercher R. B., 1973. Laboratory incubation studies of chlorophenoxyacetic acids in chernozemic soils. Soil Biol. Biochem., 5 (3), 333-337.

Fournier J. C., Codaccioni P., Soulas G., 1981. Soil adaptation to 2,4-D in relation to the application rates and the metabolic behaviour of the degrading microflora. Chemosphere, $10(8), 977-984$.

Haider K., Martin J. P., Filip Z., 1975. Humus biochemistry, pp. 195-244. In "Soil Biochemistry"' Vol. 4 ; E. A. PAUL et A. D. Mc Laren ed. Marcel Dekker, Inc., New York.

Huber S. J., Poschenrieder G., Wallnofer P. R., 1980. Einfluss von Pflanzenbehandlungsmitteln und deren Metaboliten auf Wachstum und Atmung einiger Bodenmikroorganismen. Z. Pflkrankh. Pflaanzenschutz, 87 (9), 533-545.
Kassim G., Martin J. P., Haider K., 1981. Incorporation of a wide variety of organic substrate carbon into soil biomass as estimated by the fumigation procedure. Soil Sci. Soc. Am. J., 45, 1106-1112.

Kunc F., Rybarova J., 1983. Mineralization of carbon atoms of ${ }^{14} \mathrm{C}$ 2,4-D side chain and degradation ability of bacteria in soil. Soil Biol. Biochem., 15 (2), 141-144.

Martin J. P., Haider K., Linhares L. F., 1979. Decomposition and stabilization of ring ${ }^{14} \mathrm{C}$ labeled catechol in soil. Soil Sci. Soc. Am. $J ., 43,100-104$.

Minard R. D., Liu S. Y., Bollag J. M., 1981. Oligomers and quinones from 2,4-dichlorophenol. J. agric. Food Chem., 29 (2), 250-253.

Narang A. S., Vernoy C. A., Eadon G. A., 1983. Evaluation of Nielsen-Kryger steam distillation technique for recovery of phenols from soil. J. Assoc. off. anal. Chem., 66 (6), 1330-1334.

Smith A., 1985. Identification of 2,4-dichloroanisole and 2,4-dichlorophenol as soil degradation products of ring-labelled $\left({ }^{14} \mathrm{C}\right) 2,4-\mathrm{D}$. Bull. environ. Contam. Toxicol., 34, 150-157.

Siuda F. J., De Bernardis J. F., 1973. Naturally occurring halogenated organic compounds. Lloydia, 36, 107-143.

Soulas G., Codaccioni P., Fournier J. C., 1983. Effect of crosstreatment on the subsequent breakdown of 2,4-D, MCPA and 2,4,5$\mathrm{T}$ in the soil. Behaviour of the degrading microbial populations. Chemosphere, $12(7 / 8), 1101-1106$.

Soulas G., Chaussod R., Verguet A., 1984. Chloroform fumigation technique as a means of determining the size of specialized soil microbial populations. Application to pesticide-degrading microorganisms. Soil Biol. Biochem., 16 (5), 497-501.

Stevenson F. J., 1972. Organic matter reactions involving herbicides in soil. J. environ. Qual., 1 (4), 333-343.

Stott D. E., Martin J. P., Focht D. D., Haider K., 1983. Biodegradation stabilization in humus, and incorporation into soil biomass of 2,4-D and chlorocatechol carbon. Soil Sci. Soc. Am. J., 47 (1), 66-70. 